Research Article

\title{
Personality Trait Detection Based on ASM Localization and Deep Learning
}

\author{
JinFeng Fu ${ }^{1}$ and Hongli Zhang $\mathbb{D}^{2}$ \\ ${ }^{1}$ School of Marxism, Heilongjiang University of Science and Technology, Harbin 150022, China \\ ${ }^{2}$ Department of Mathematics and Computer Science, Tongling University, Anhui, Tongling 244061, China
}

Correspondence should be addressed to Hongli Zhang; zhl0@tlu.edu.cn

Received 17 July 2021; Revised 2 August 2021; Accepted 14 August 2021; Published 21 August 2021

Academic Editor: Bai Yuan Ding

Copyright (c) 2021 JinFeng Fu and Hongli Zhang. This is an open access article distributed under the Creative Commons Attribution License, which permits unrestricted use, distribution, and reproduction in any medium, provided the original work is properly cited.

\begin{abstract}
Global competition is the competition of human resources, the social demand for high-quality talents is increasing, and the demand for all kinds of talents is increasing. Therefore, how to scientifically and efficiently complete the preliminary screening of college students' mental health, so as to provide services for them, has become an important task. In order to solve the above problems, by combining the relevant professional knowledge of psychology, statistics, image processing, and artificial intelligence technology, a personality trait detection method based on active shape model (ASM) localization and deep learning is proposed. Firstly, the traditional ASM algorithm is improved and applied to facial feature point location, which provides training basis for further deep learning. It mainly includes three aspects of improvement: (1) 2D texture model based on Gabor wavelet and gradient features; (2) new multiresolution pyramid decomposition method; and (3) improved multiresolution pyramid search strategy. Secondly, the deep belief network model is used to train and classify the students' four personality traits and facial features, so as to dig out the relationship between the four personality traits and facial features. The experimental results show that the localization effect of the improved ASM algorithm is obviously better than that of the traditional algorithm, and the classifier after learning and training has a good effect in analyzing the four personality traits.
\end{abstract}

\section{Introduction}

In essence, global competition is the competition of human resources. Therefore, the social demand for high-quality talents is increasing, and the demand for all kinds of talents is increasing. Therefore, how to scientifically and efficiently complete the preliminary screening of college students' mental health, so as to provide services for them, has become an important task.

As a cross-discipline, management science and engineering include many research fields [1-5]. In order to classify the personality characteristics of talents accurately and quickly, management science and engineering need to combine relevant professional knowledge such as psychology, statistics, image processing, and artificial intelligence technology to establish a personality classification tree based on facial features to achieve efficient personality trait classification. Thus, we can understand the potential psychological problems of college students at the first time, effectively improve their mental health, and help quickly find suitable roles according to their personality.

Face can best reflect a person's immediate mental outlook; face can express emotion and can better reflect a person's character and life [6-8]. The results show that, just like adults, even 3-year-olds tend to judge their personality traits, such as credibility and ability, by their faces, and children show surprising consistency with adults in judgment. With the increase of age, the tendency to judge people by their appearance also develops, and the older children are, the more similar their facial judgments are to adults. These psychological studies show that people's personality and even in a certain aspect of the ability and social behavior. The character of a character refers to the relatively stable and core psychological characteristics of his attitude and behavior in 
the face of real things. It is a kind of personality characteristic with the highest correlation with society, which is closely related to facial features [9-12]. The features of human face can be regarded as the distance between facial contours, eyebrows, eyes, nose, mouth, and other feature points, including the distance between eyebrows and eyes, eyes and nose, nose and mouth, even the distance between the corner of the eye and the inner canthus, the left and right pupil, the corner of the left and right mouth, and so on. How to associate human character with complex facial features and find the relationship between them is the primary problem to be solved in this study.

In psychology, there are many ways to measure a person's personality characteristics, including psychological counseling, psychological scale testing, and other means $[13,14]$. Different from the existing methods, on the basis of traditional personality test, this study combines face recognition technology and data mining technology to quickly and accurately find the relationship between facial features and personality traits and establish a student personality classification tree based on facial features. The main contributions of this paper are as follows:

(1) The traditional ASM algorithm is improved in three aspects, and the improved algorithm is applied to the facial feature point location, which solves the shortcomings of the traditional ASM algorithm, such as inaccurate location, low efficiency, and easy to fall into local minimum.

(2) With the help of the characteristics of deep learning unsupervised learning classification, the sample data are trained by deep belief network model to obtain a classifier based on four personality traits and facial features.

(3) Finally, through the comparative analysis of the classification results of psychological personality test, it verifies the feasibility and effectiveness of this method and provides a scientific basis for college teachers to make a reasonable psychological counseling plan.

The rest of the paper is organized as follows. In Section 2, a literature review is studied in detail, while Section 3 provides the detailed methodology. Section 4 provides detailed results and discussion. Finally, the paper is concluded in Section 5.

\section{Literature Review}

Firstly, face feature point location technology is the main content of this study. According to the number of feature points, facial feature point location methods can be divided into two categories: local feature point location method and global feature point location method.

The local feature point location method can only locate a small number of feature points at a time, and it is often used to locate local facial features. In [15], Akash et al. proposed a method to locate the center of pupil and mouth based on $\mathrm{YCbCr}$ color space distribution. The advantage of this method is that the location of human face can be determined quickly by determining the position of three points.

Different from the local feature point location method, the global feature point location method can locate many feature points at one time. The more popular methods include active shape model (ASM) and active appearance model (AAM). In [16], Wang et al. proposed to recognize human emotion through facial images. ASM method is used to reconstruct facial shape, extract facial feature points, and then use radial basis function network to recognize facial expression. In [16], Wang et al. used ASM in the driving fatigue detection system and introduced the average synthetic accurate filter algorithm to improve the positioning accuracy and attitude adaptability of the eye.

Secondly, for deep learning technology. The acquisition of learners' personality characteristics is an important prerequisite for the realization of student-centered accurate and personalized teaching, and learning behavior is an important basis for the analysis of learners' personality characteristics. Deep learning is a kind of machine learning. The main step of machine learning is to obtain the initial data through the sensor, and after the data is preprocessed, the data features are extracted and selected. After feature expression, the process of reasoning, prediction, and recognition can be carried out.

In [17], Zhao et al. used Rapid Miner data mining tools to explore the recognition effects of decision tree, naive Bayes, and support vector machine on five personality traits. The results show that the accuracy of the decision tree algorithm for the recognition of personality traits is higher than that of the other two algorithms, and the comprehensive recognition effect of the big five personality traits is the best. The sensitivity of recognition of different personality traits is different. The sensitivity of conscientious personality trait type is the highest, while that of neurotic personality trait is the lowest.

As a classical neural network learning method, deep belief network learning method can be regarded as a neural network composed of multiple restricted Boltzmann machine layers. Among them, the network is limited to two layers, namely, the data visual layer and the hidden layer. And only there is a connection between layers, and the nodes in the network exist independently. Among them, the data in the hidden layer can be trained to obtain the data in the data visual layer.

\section{Methodology}

3.1. Student Face Feature Point Localization Algorithm Based on Improved ASM. In the process of ASM modeling, it is very important to calibrate the feature points of the training sample image. The accuracy of feature point calibration of sample image is directly related to the representativeness of the shape model. Figure 1 shows an example of how this article has marked a set of pictures. Each image is manually calibrated with 32 feature points.

3.1.1. 2D Texture Model Based on Gabor Wavelet and Gradient Feature. The traditional ASM algorithm uses 1D 


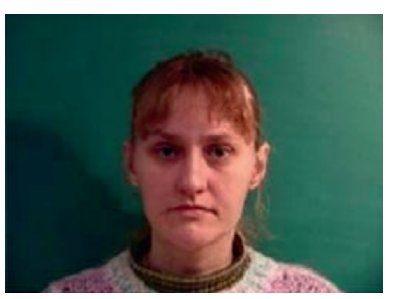

Training sample 1

(a)

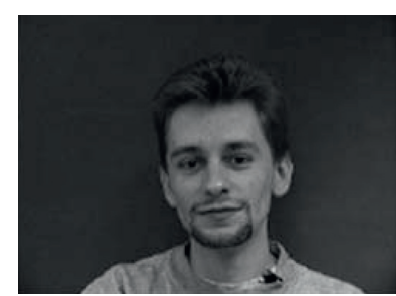

Training sample 2

(b)

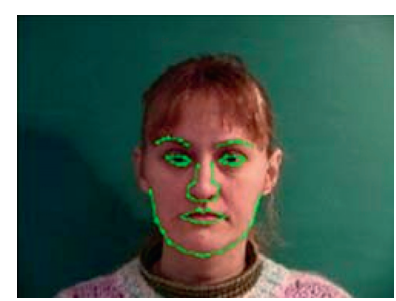

Tagged sample 1

(c)

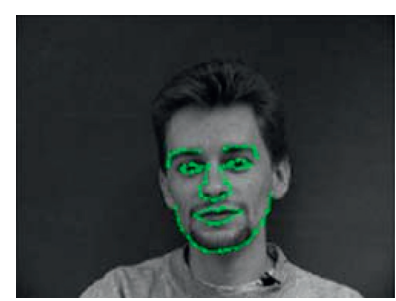

Tagged sample 2

(d)

Figure 1: A set of tagged images. (a) Training sample 1. (b) Training sample 2. (c) Tagged sample 1. (d) Tagged sample 2.

gray information near the feature points as matching features and takes the distance between the gray value vector at the target candidate points and the local gray model at the mark points as the similarity measure between the candidate points and the mark points [18-21]. In the search process, only the low-frequency information of the face is used. The low-frequency components mainly describe the global features of the object, while the high-frequency components mainly describe the local details. Therefore, the calculation amount of this algorithm is small, but the positioning is not very accurate [22-24].

In order to solve the above problems caused by the $1 \mathrm{D}$ texture model, we introduce a new method to build the local model of feature points, that is, to build a $2 \mathrm{D}$ local texture model for each feature point, as shown in Figure 2.

As shown in Figure 2, the current feature point can reach its target position $P 1$, so the positioning error will be reduced. For each marked feature point, select a rectangular region with side length of $m \times n$, and the current feature point is the center of the rectangular region. By extracting the Gabor feature or gradient feature of each point in the rectangular region, the corresponding Gabor texture model or gradient texture model of the feature point can be obtained [25]. Since there are $N$ face images in the training set, for each feature point, $N$ rectangular regions can be obtained; that is, $N$ 2D texture models can be obtained. Each $2 \mathrm{D}$ texture model is arranged row by row into a long vector, and all the $2 \mathrm{D}$ texture models form a matrix with $N$ rows and $m \times n$ columns.

Gabor wavelet transform can extract spatial frequencies and local features in multiple directions in the local region of the image [26]. Therefore, Gabor feature description of human face can achieve a good recognition effect. In image processing and computer vision, two-dimensional Gabor wavelet is mainly used. The two-dimensional Gabor wavelet is expressed as follows:

$$
W(x, y ; \theta, \lambda, \varphi, \sigma, \gamma)=\exp \left(-\frac{x^{\prime 2}+\gamma^{2} y^{\prime 2}}{2 \sigma^{2}}\right) \cos \left(2 \pi \frac{x^{\prime}}{\lambda}+\varphi\right)
$$

where $(x, y)$ represents the pixel position in the time domain, $x^{\prime}=x \cos \theta+y \sin \theta, \quad y^{\prime}=-x \sin \theta+y \cos \theta . \quad \theta$ denotes the direction parameter, $\lambda$ denotes the wavelength parameter of the triangle cosine function, $\varphi$ denotes the phase offset parameter, $\sigma$ denotes the standard deviation of

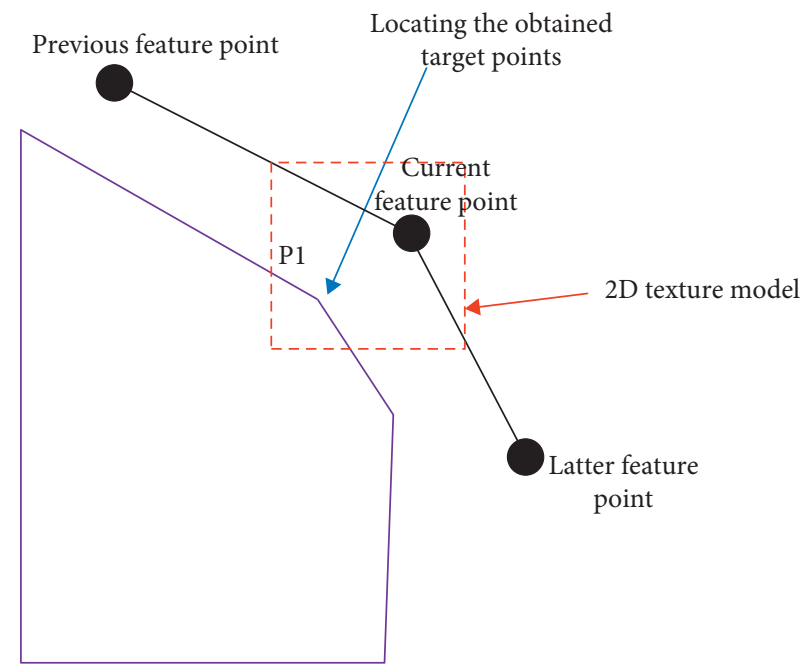

Figure 2: 2D local texture model.

the Gaussian function, and $\gamma$ denotes the length-width ratio of the Gabor kernel. Two-dimensional Gabor wavelets with different shapes can be generated by selecting different parameters.

We use 40 Gabor wavelet functions combined with 5 frequencies and 8 directions to carry out the convolution operation on a point in the image, and 40 complex coefficients can be obtained, which are called the Gabor jet vector of the point. The 40 Gabor wavelet kernel functions adopted are shown in Figure 3.

Traditional ASM algorithm is to establish a local gray statistical feature texture model in a small area near the control point (sampling in the normal direction). Studies show that using gray scale as a local feature requires a lot of training sample images to obtain sufficient statistical information, and gray scale feature without gradient as a local feature has better robustness [27]. Since gray scale features are sensitive to illumination, in order to reduce the impact of illumination on positioning accuracy, and due to the limited number of training sample sets, a texture model based on $2 \mathrm{D}$ gradient features is established for each feature point of the highest level and sub-high-level pyramid subgraph of each image.

The 2D gradient texture model of each marker is generated by the following two steps: convolution and normalization. 

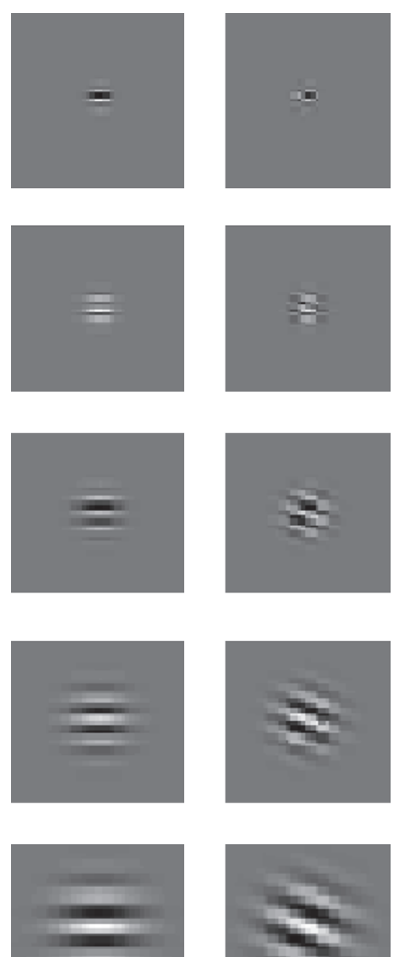
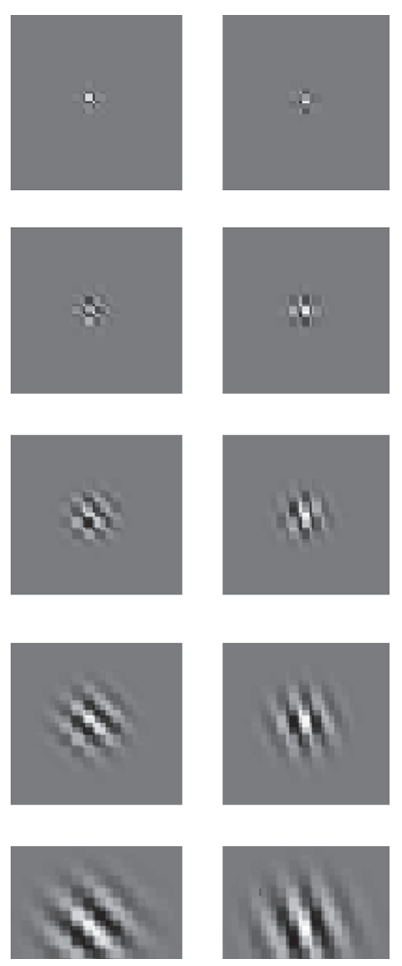
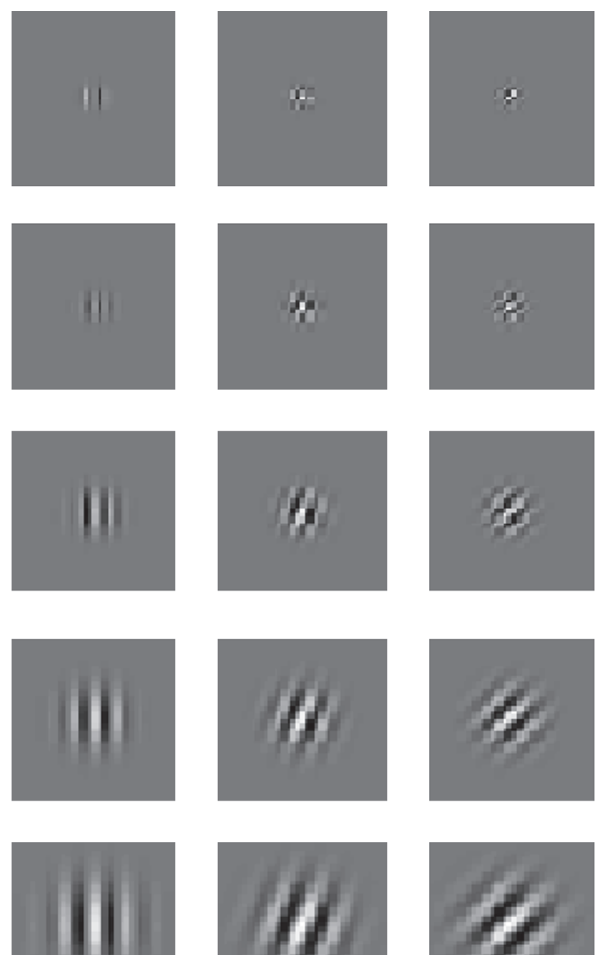
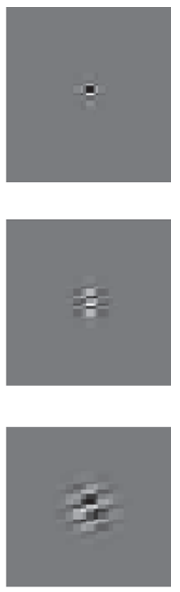

Figure 3: Schematic diagram of 40 Gabor nuclei.
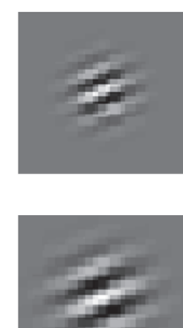

(1) Convolution. In this step, a $3 * 3$ convolution mask is used to produce a texture matrix for each pixel of the rectangular region (size $m \times n)$ around the marker. Each element of the texture matrix corresponds to a pixel convolution value. The value of the element is a representation of some image feature of the pixel. This feature is determined by the convolution mask.

Because the gradient features of feature points are to be extracted in this paper, the gradient mask is used, and the gradient mask matrix is

$$
\mathbf{A}=\left[\begin{array}{ccc}
0 & 0 & 0 \\
0 & 2 & -1 \\
0 & -1 & 0
\end{array}\right] .
$$

By convolution, the gradient texture matrix of the feature point can be obtained, which is $2 \mathrm{D}$.

(2) Normalization Eliminates. In order to eliminate the influence of illumination and contrast, the gradient texture matrix also needs to be normalized. Each element of the gradient texture matrix is normalized by dividing the absolute value of all elements in the matrix.

By doing the same operation for the same marked point of each training sample image, $N 2 \mathrm{D}$ gradient texture matrices can be obtained. Here, each $2 \mathrm{D}$ gradient texture matrix is still arranged into a long vector by row, and all the long vectors form a matrix, which has $N$ rows and $m \times n$ columns. At this time, the mean and covariance matrices of $2 \mathrm{D}$ gradient texture matrix are obtained, and the mean and covariance matrices are used as the matching features of this point.
It is proved that using gradient information instead of gray information to build local feature texture model can effectively improve the matching accuracy and reduce the number of sample images to be trained.

\subsubsection{A New Multiresolution Pyramid Decomposition} Method. In the training and searching phase of ASM under the framework of multiresolution analysis, the traditional ASM method first performs Gaussian filtering on the initial sample image and then samples the filtered image at intervals to get the pyramid image of the training sample. The resolution of these images decreases in turn, and the scale of the latter image is $1 \max 2$ of the previous one. The initial sample image is Level 0 (layer 1). With the gradual decrease of resolution, the pyramid subimage obtained by this method contains less and less detail features, which is not conducive to the extraction of rich texture features, thus affecting the accuracy of feature point location.

In this paper, the size of the training picture and the test picture is $480 * 640$. The pyramid subimage is obtained by using db1 wavelet to decompose the sample image with 3layer wavelet, as shown in Figure 4.

The sample image is decomposed by Gaussian filter of original ASM algorithm in three layers, and the pyramid subgraph obtained is shown in Figure 5.

It can be clearly seen from the experimental results of the above two graphs that, with the gradual reduction of resolution, the pyramid subgraph obtained by wavelet decomposition contains more detailed information than that obtained by original ASM. The rich detailed information is beneficial to the extraction of rich local texture features and 


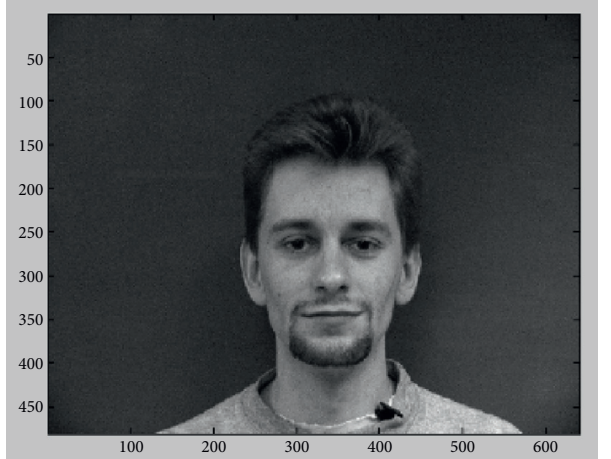

zero-order subgraph

(a)

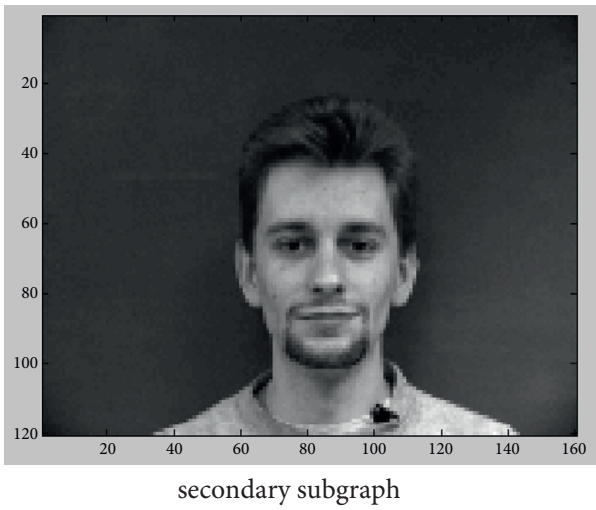

(c)

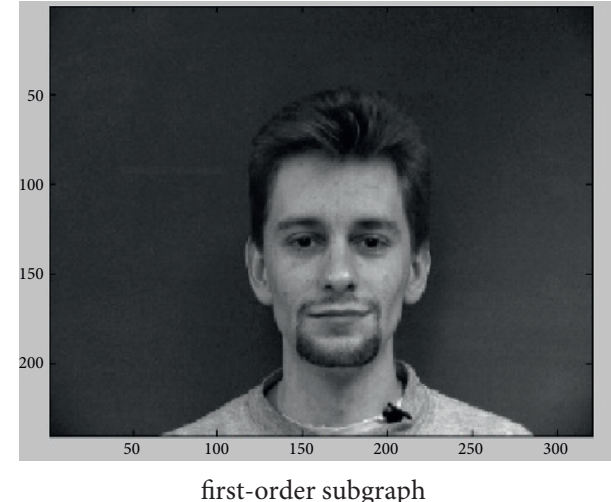

(b)

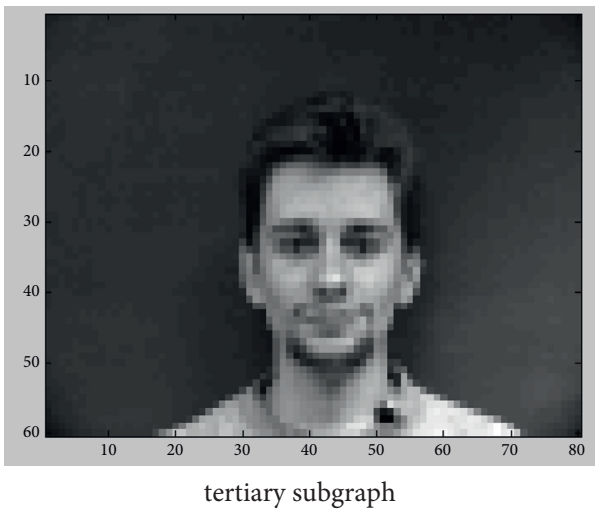

(d)

FIgURe 4: Pyramid subgraph obtained by wavelet decomposition. (a) Zero-order subgraph. (b) First-order subgraph. (c) Secondary subgraph. (d) Tertiary subgraph.

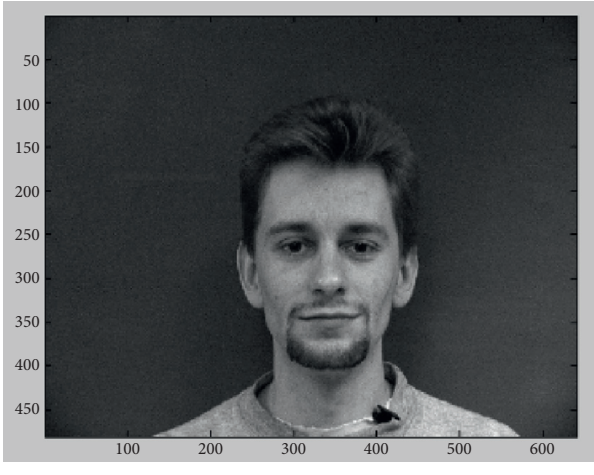

zero-order subgraph

(a)

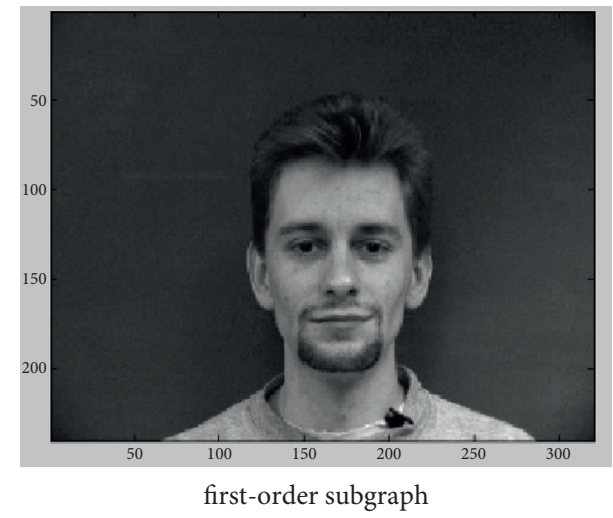

(b)

FIGURE 5: Continued. 


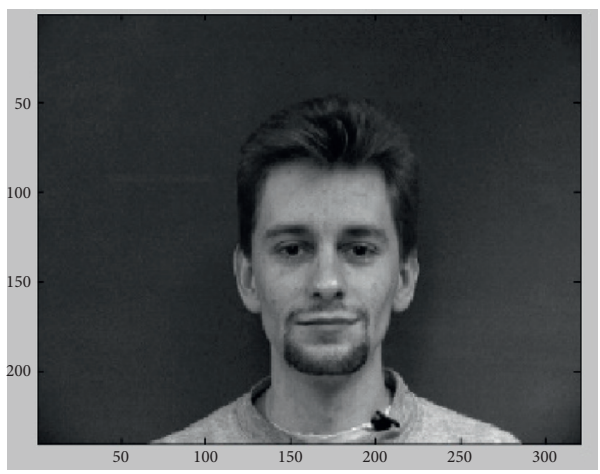

secondary subgraph

(c)

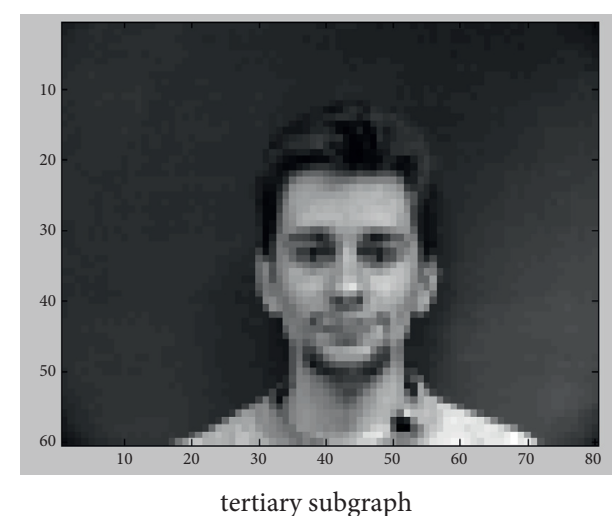

(d)

Figure 5: Pyramid subgraph obtained by Gaussian filter decomposition. (a) Zero-order subgraph. (b) First-order subgraph. (c) Secondary subgraph. (d) Tertiary subgraph.

the establishment of reliable texture model, so as to improve the accuracy of feature point localization.

\subsubsection{Improved Multiresolution Pyramid Search Strategy.} In the traditional ASM algorithm, when multiresolution search is carried out, the search length of different layers is the same; that is, the same number of pixels are sampled for each feature point in each contour texture model of each layer. In this paper, the first layer (lowest layer) of the multiresolution pyramid is set as the original image, and the second layer (second layer) and the third layer (highest layer) are set as the pyramid subgraph obtained by wavelet decomposition.

This paper proposes to adaptively change the length of $2 \mathrm{D}$ texture search rectangle according to the different number of pyramid decomposition layers. Under low resolution, because some details of the image are ignored and the feature points are far away from the actual target position, a larger search range can be set, so as to obtain an ideal matching result for the overall contour. Correspondingly, at a higher resolution, because the feature points are close to the target position, the search range can be appropriately reduced, so as to obtain a relatively ideal local matching result. The edge length of $2 \mathrm{D}$ texture search rectangle adaptively decreases as the number of layers of multiresolution pyramid decreases. In this paper, we set the length of the search rectangle on the third layer to be $L$, the second layer to be $1 / 2$, and the first layer to be $L / 4$. The efficiency of this algorithm is improved because the computation is small at lower resolution.

3.2. Personality Trait Analysis Based on Deep Belief Network Model and Facial Features. In order to explore the potential relationship between the four personality traits and facial features, we trained and learned the sample data through the deep belief network model, in order to obtain a trained classifier based on the four personality traits and facial features of students.

3.2.1. Deep Belief Network Model. In the field of computer vision, deep learning technology has shown its excellent performance. However, sometimes it is difficult to learn directly from the whole image using standard deep learning techniques, especially with complex data such as high-definition images. As an efficient deep learning algorithm, Deep Belief Networks (DBN) have gradually developed into a mainstream technology direction. Based on the principle of statistical mechanics, a random neural network Boltzmann machine model is generated, including a hidden layer and a visible layer, as shown in Figure 6.

On this basis, the principle architecture of multilayer restricted Boltzmann machine (RBM) is proposed, as shown in Figure 7, where $\mathbf{a}=\left(a_{1}, a_{2}, \ldots, a_{n_{v}}\right)^{T} \in R^{n_{v}}$ is the bias vector of the visible layer, $\mathbf{b}=\left(b_{1}, b_{2}, \ldots, b_{n_{h}}\right)^{T} \in R^{n_{h}}$ is the bias vector of the hidden layer, and $\mathbf{W}=\left(w_{i, j}\right) \in R^{n_{h} \times n_{v}}$ is the weight matrix between the hidden layer and the visible layer. Through the generative stacking technology, the depth belief network is finally produced by multiple restricted Boltzmann machines.

The constrained Boltzmann machine introduces a series of related probability distribution functions through the energy function. For the state vector $(\mathbf{v}, \mathbf{h})$ of a given set of neurons, its energy function can be expressed as

$$
E(\mathbf{v}, \mathbf{h} \mid \boldsymbol{\theta})=-\sum_{i=1}^{n_{v}} a_{i} v_{i}-\sum_{j=1}^{n_{h}} b_{j} h_{j}-\sum_{i=1}^{n_{v}} \sum_{j=1}^{n_{h}} h_{j} w_{i, j} v_{i},
$$

where $\mathbf{v}$ represents the state vector of neurons in the visible layer, and $\mathbf{h}$ represents the state vector of neurons in the hidden layer. $n_{v}$ is the total number of all neurons in the visible layer, and $n_{h}$ is the total number of all neurons in the hidden layer. $\theta=\left\{a_{i}, b_{j}, w_{i, j}\right\}$ is the regulator limiting the Boltzmann machine architecture. 


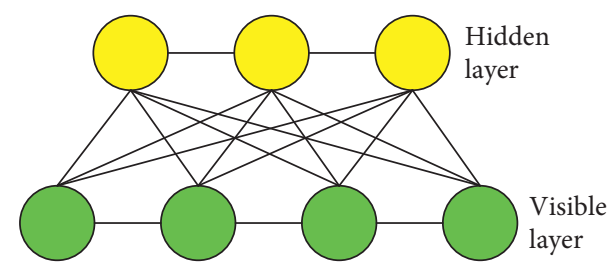

FIgURE 6: Boltzmann machine model.

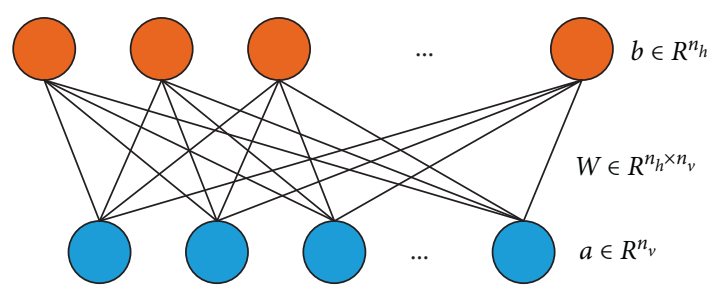

Figure 7: RBM model

Through the energy function defined in equation (3) above, the joint probability distribution of state $(\mathbf{v}, \mathbf{h})$ can be obtained as shown in

$$
\begin{aligned}
P(\mathbf{v}, \mathbf{h} \mid \boldsymbol{\theta}) & =\frac{1}{Z(\boldsymbol{\theta})} e^{-E(\mathbf{v}, \mathbf{h} \mid \boldsymbol{\theta})}, \\
Z(\boldsymbol{\theta}) & =\sum_{\nu, h} e^{-E(\mathbf{v}, \mathbf{h} \mid \boldsymbol{\theta})} .
\end{aligned}
$$

In the formula, $Z(\theta)$ represents the normalized parameter. Let $p(\mathbf{v} \mid \theta)$ be the probability distribution of the visible layer vector $\mathbf{v}$; then $p(\mathbf{v} \mid \theta)$ can be calculated by the edge distribution of $P(\mathbf{v}, \mathbf{h} \mid \theta)$.

$$
\begin{aligned}
P(\mathbf{v} \mid \boldsymbol{\theta}) & =\sum_{h} P(\mathbf{v}, \mathbf{h} \mid \boldsymbol{\theta}) \\
& =\frac{1}{Z(\boldsymbol{\theta})} \sum_{h} e^{-E(\mathbf{v}, \mathbf{h} \mid \boldsymbol{\theta})} .
\end{aligned}
$$

In the same way, we can get the probability distribution $p(\mathbf{h} \mid \theta)$ of the hidden layer vector $\mathbf{h}$.

$$
\begin{aligned}
P(\mathbf{h} \mid \boldsymbol{\theta}) & =\sum_{v} P(\mathbf{v}, \mathbf{h} \mid \boldsymbol{\theta}) \\
& =\frac{1}{Z(\boldsymbol{\theta})} \sum_{v} e^{-E(\mathbf{v}, \mathbf{h} \mid \boldsymbol{\theta})} .
\end{aligned}
$$

By analyzing equations (6) and (7), it can be seen that in order to get $p(\mathbf{v}, \mid \theta)$ and $p(\mathbf{h} \mid \theta)$, the key step is to calculate the normalized parameter $Z(\theta)$. However, according to the definition of $Z(\theta)$, its computational complexity is relatively high. Due to the special principle of limiting Boltzmann machine model (the visible layer and the hidden layer are conditionally independent), when the state of all neurons in the visible layer is known, the probability of activation of a neural unit in the hidden layer can be calculated by

$$
P\left(h_{j}=1 \mid \mathbf{v}, \boldsymbol{\theta}\right)=\sigma\left(b_{j}+\sum_{i} v_{i} w_{i j}\right),
$$

where $\sigma(\cdot)$ represents the Sigmoid activation function.

Because there is no connection between all neural nodes in the same layer, the relationship between the values of all neural nodes in the same layer and the values of a single node is as follows:

$$
\begin{aligned}
& P(\mathbf{h} \mid \mathbf{v})=\prod_{j-1}^{n_{h}} P\left(h_{j} \mid v\right), \\
& P(\mathbf{v} \mid \mathbf{h})=\prod_{i-1}^{n_{v}} P\left(v_{i} \mid h\right) .
\end{aligned}
$$

The random gradient algorithm is usually used to find the maximum value in order to obtain the optimal adjustment factor in the network. The DBN model is shown in Figure 8.

3.2.2. Feature Classification Process. DBN training process is generally divided into two steps: (1) pretraining stage and (2) fine-tuning stage. The loss function required in the finetuning stage is shown in

$$
L(x, y)=\|x-y\|_{2}^{2},
$$

where the symbol $\|\cdot\|_{2}$ represents the 2 norm of reconstruction error, $x$ represents input data, and $y$ represents reconstructed data.

The data comes from the face images of the tested students in the sample set, which includes a training sample set, a test sample set, and a text document of the training sample classification information. The feature classification process is shown in Figure 9.

Step 1: divide personality traits into three categories (e.g., low agreeableness, moderate agreeableness, and high agreeableness), input 32 feature information extracted based on active model, respectively, and form 32 feature vectors representing feature information.

Step 2: classify the data samples. The data in the data sample is divided into two folders, the training sample set and the test sample set, and all the sample data in the training sample set are labeled by categories to form a text document, which can be used in the later learning and training of image features.

Step 3: sample training. According to the deep belief network structure model, this paper adopts the deep belief network structure model containing five layers of RBM to train the training samples provided.

Step 4: test the sample. After training the training samples with the deep confidence network, the relevant weights and biases of each layer of DBN can be obtained. Then, the relevant classification results can be obtained by testing the test samples. 


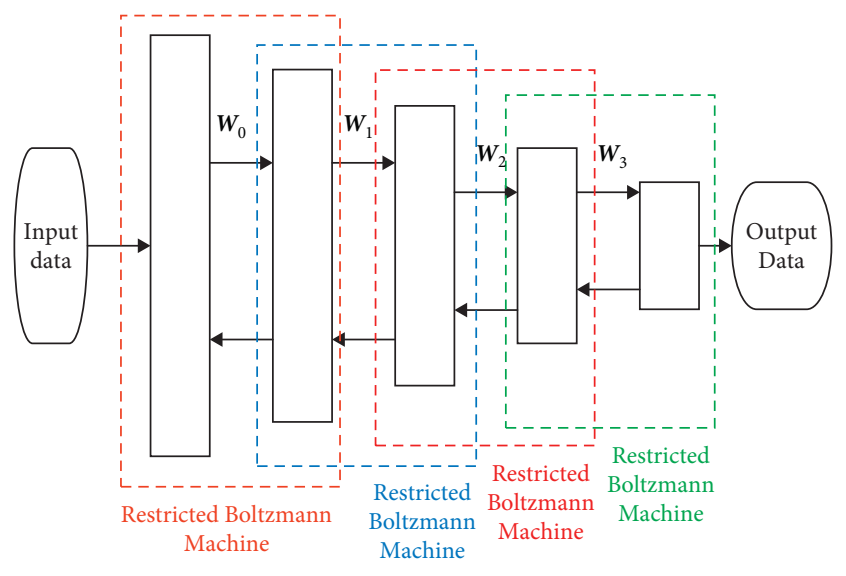

Figure 8: DBN model.

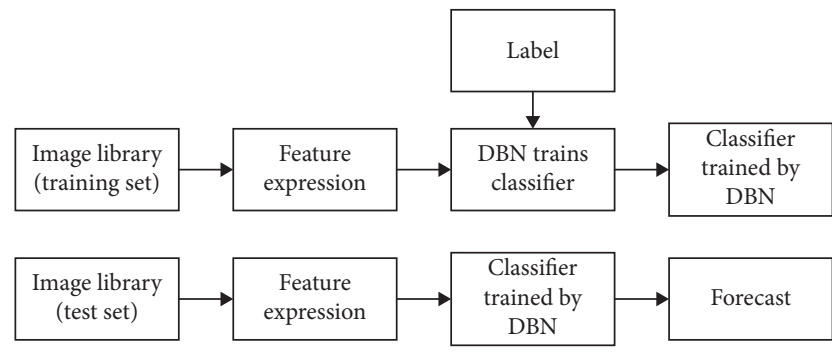

Figure 9: Feature classification process.

The classifiers of extroversion, openness, agreeableness, and conscientiousness can be established after the four personality traits are processed, respectively, according to the above steps.

\section{Experimental Results and Analysis}

4.1. Experimental Environment. The experiment is carried out on IMM face database [28-33], and the experimental simulation tool is MATLAB7.8. IMM face database consists of 240 images of six faces of 40 different people, all without glasses. The image size is $640 * 480.120$ face images are selected from the library as the training set and 80 as the test images. In order to simplify the problem, the images used for training and testing were all neutral expressions of positive faces, and the illumination was relatively uniform. The feature points are marked on the external contour of the face and the edges of each facial organ. Each training image was calibrated with 58 feature points. After repeated tests, this paper sets the 2D texture rectangular search side lengths of the third layer, the second layer, and the first layer as 16,8 , and 4 , respectively.

4.2. Performance Evaluation Indicators. In order to test the performance of the improved ASM algorithm, two functions are introduced to evaluate its performance. The first is the average error function:

$$
E_{\mathrm{ave}}=\frac{1}{N} \times \frac{1}{n} \sum_{i=1}^{N} \sum_{j=1}^{n} \operatorname{abs}(x(i, j)-\operatorname{pos}(i, j)),
$$

where $N$ is the total number of test images and $n$ is the number of feature points marked. $x(i, j)$ is the coordinate of the $j$ th feature point marked by the $i$ th subimage, and pos $(i, j)$ is the coordinate [34] of the target point located by ASM algorithm.

The other is the time function of the average cost:

$$
\mathrm{T}_{\text {ave }}=\frac{1}{N} \sum_{i=1}^{N} t_{i}
$$

where $t_{i}$ is the time it takes to process each picture $[35,36]$.

4.3. Comparison of Face Feature Points Location Results. The effect of improved ASM and traditional ASM for face feature point localization is shown in Figures 10 and 11, respectively.

Under the premise of 50 iterations, the average error between the improved ASM and the traditional ASM is compared, and the result is shown in Figure 12.

It can be seen from the experimental results that the improved ASM algorithm can more accurately locate the key face feature points and improve the positioning accuracy of face feature points. The performance comparison results between improved ASM and traditional ASM are shown in Table 1.

By comparing the results, it can be seen that the proposed method is more accurate than the original algorithm. The improved algorithm is less efficient than the original one because it builds a $2 \mathrm{D}$ texture model with a large amount of computation for each feature point and extracts Gabor features with a large amount of computation for the lowest pyramid subgraph of each feature point. However, it can be seen from the experimental results that the positioning accuracy of the proposed algorithm is more than doubled compared with the original algorithm, while the processing time is not doubled. Therefore, the shortcoming of the algorithm efficiency decline is acceptable.

4.4. Classifier Test. Through the training samples, we can get four classifiers after DBN training: extroversion, openness, agreeableness, and rigor.

The DBN model is compared with the convolutional neural network model, and the results of recognition accuracy and consumption time are shown in Table 2 and Figure 13.

As can be seen from Table 2 and Figure 13, the classifier trained by DBN is used to test four kinds of personality traits of the test sample. The accuracy of extroversion is $77.95 \%$, the accuracy of openness is $81.16 \%$, the accuracy of agreeableness is $90.63 \%$, and the accuracy of rigor is $91.42 \%$, totaling $85.29 \%$, which is higher than that of the classifier trained by convolutional neural network model.

Thus, it can be seen that the classifier trained by DBN has a good effect on the prediction results of the four trait tests on the premise of providing facial features. 


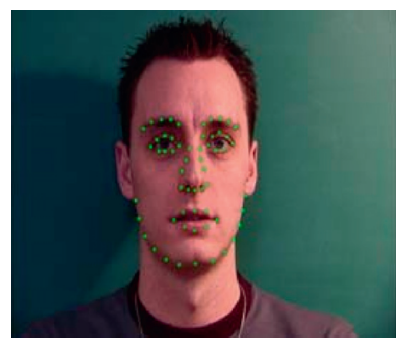

feature point location result 1

(a)

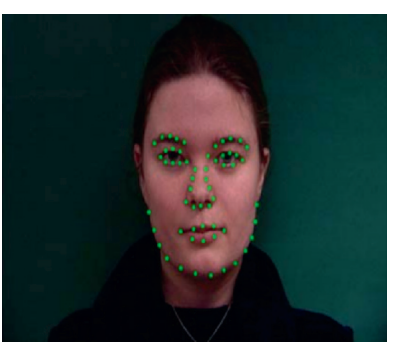

feature point location result 2

(b)

FIGURE 10: Effect of face feature point location using improved ASM. (a) Feature point location result 1. (b) Feature point location result 2.

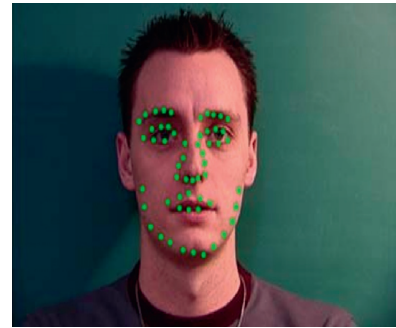

feature point location result 1

(a)

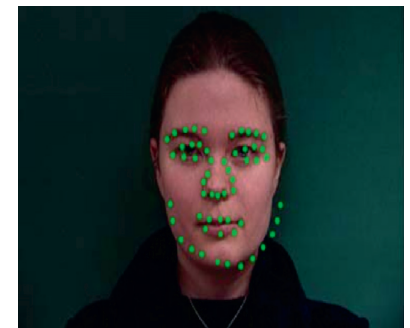

feature point location result 2

(b)

FIGURE 11: Effect of face feature point location using traditional ASM. (a) Feature point location result 1. (b) Feature point location result 2.

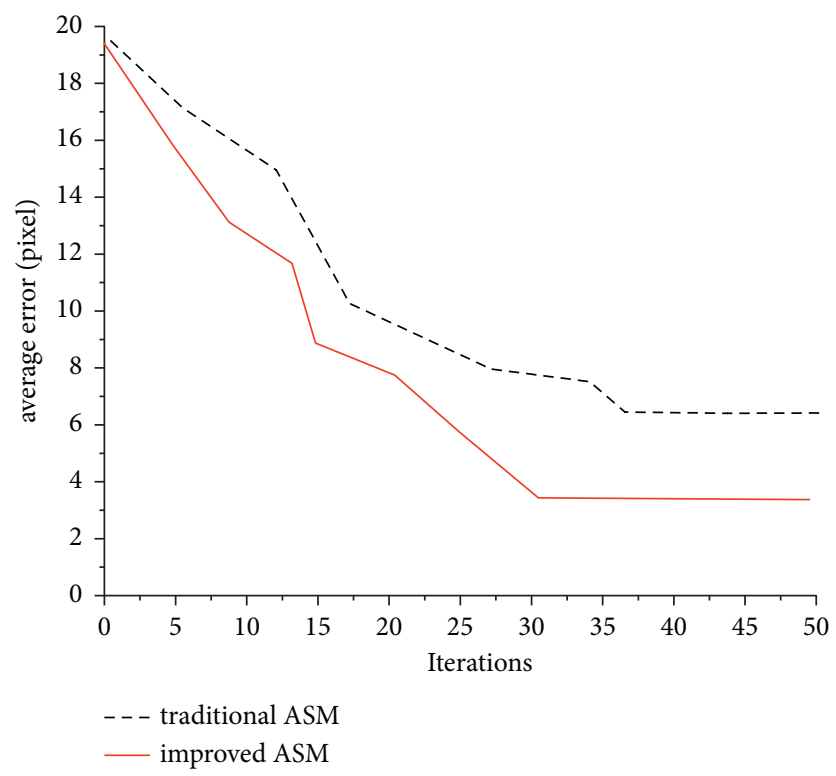

FIGURE 12: Comparison of average error between improved ASM and traditional ASM.

TABLE 1: Comparison of the performance of improved and traditional ASM.

\begin{tabular}{lcc}
\hline Objective function & Improved ASM & Traditional ASM \\
\hline$E_{\text {ave }}$ (pixels) & 3.16 & 6.34 \\
$T_{\text {ave }}(\mathrm{s})$ & 4.67 & 3.09 \\
\hline
\end{tabular}


TABLE 2: Comparison of the accuracy of two classifiers.

\begin{tabular}{|c|c|c|}
\hline Machine learning approach & Classifier & Accuracy (\%) \\
\hline \multirow{4}{*}{ Convolution neural network model } & Extroversion openness & $\begin{array}{l}73.88 \\
78.57\end{array}$ \\
\hline & Agreeableness & 85.73 \\
\hline & Rigor & 87.74 \\
\hline & Total & 81.48 \\
\hline \multirow{5}{*}{ BP neural network model } & \multirow{2}{*}{ Extroversion openness } & 69.05 \\
\hline & & 73.45 \\
\hline & Agreeableness & 80.72 \\
\hline & Rigor & 85.61 \\
\hline & Total & 77.21 \\
\hline \multirow{5}{*}{ DBN model } & \multirow{2}{*}{ Extroversion openness } & 77.95 \\
\hline & & 81.16 \\
\hline & Agreeableness & 90.63 \\
\hline & Rigor & 91.42 \\
\hline & Total & 85.29 \\
\hline
\end{tabular}

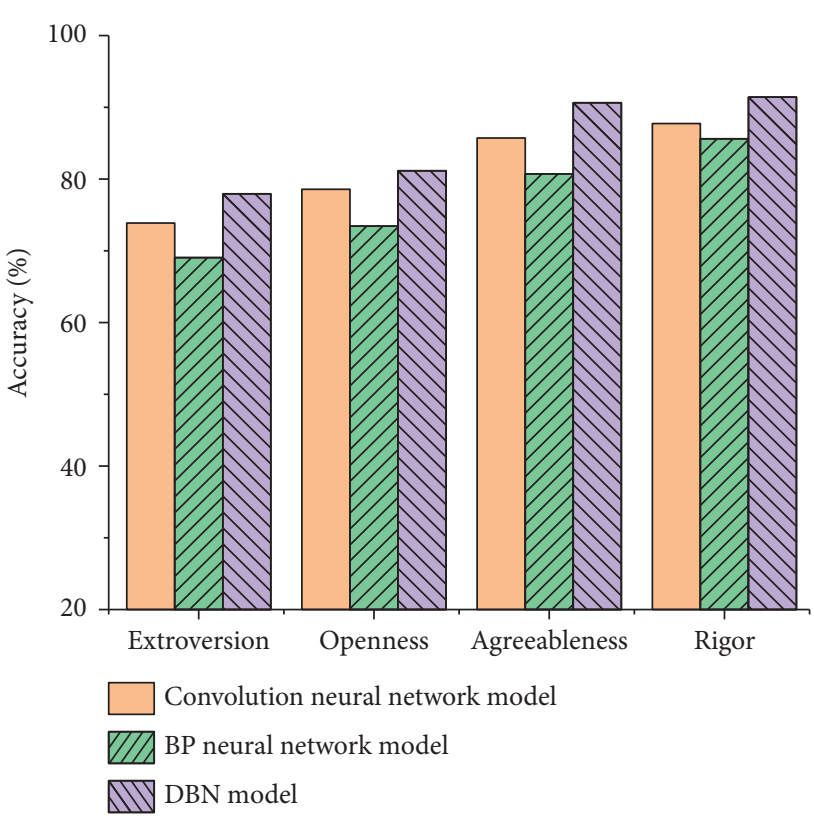

Figure 13: Comparison of the accuracy of two classifiers.

\section{Conclusion}

In this paper, a personality trait detection method based on ASM facial feature point location and DBN model is proposed. First of all, the traditional ASM algorithm is improved in three aspects, and the improved algorithm is applied to the facial feature point location, which solves the shortcomings of the traditional ASM algorithm, such as inaccurate location, low efficiency, easy to fall into local minimum, and so on. Secondly, with the help of the characteristics of deep learning unsupervised learning classification, the sample data are trained by the deep belief network model in order to get the classifier based on four personality traits and facial features. Finally, through the comparative analysis of the classification results of psychological personality test, it verifies the feasibility and effectiveness of this method and provides a scientific basis for college teachers to make a reasonable psychological counseling plan.

\section{Data Availability}

The data used in this paper are available from the corresponding author upon request.

\section{Conflicts of Interest}

The authors declare that they have no conflicts of interest.

\section{Acknowledgments}

This research was partially supported by Teaching and Research Project of Heilongjiang University of Science and Technology (no. JY20-45).

\section{References}

[1] R. Zhang, L. Lin, R. Zhang, W. Zuo, and L. Zhang, "Bit-scalable deep hashing with regularized similarity learning for image retrieval and person Re-identification," IEEE Transactions on Image Processing, vol. 24, no. 12, pp. 4766-4779, 2015.

[2] T. M. Scholtens, F. Schreuder, S. T. Ligthart, J. F. Swennenhuis, J. Greve, and L. W. M. M. Terstappen, "Automated identification of circulating tumor cells by image cytometry," Cytometry, Part A, vol. 81A, no. 2, pp. 138-148, 2012.

[3] M. Wright, C.-J. Lin, E. O’Neill, D. Cosker, and P. Johnson, "3D gesture recognition: an evaluation of user and system performance, Lecture Notes in Computer Science," in Proceedings of the International Conference on Pervasive Computing, pp. 294-313, Springer, Berlin, Heidelberg, June 2011.

[4] H. Cheng, L. Yang, and Z. Liu, "Survey on 3D hand gesture recognition," IEEE Transactions on Circuits and Systems for Video Technology, vol. 26, no. 9, pp. 1659-1673, 2015.

[5] A. Mousavian, D. Anguelov, J. Flynn, and J. Kosecka, "3d bounding box estimation using deep learning and geometry," in Proceedings of the IEEE Conference on Computer Vision and Pattern Recognition, pp. 7074-7082, IEEE, San Juan, PR, USA, June 2017.

[6] K. Sun, B. Xiao, D. Liu, and J. Wang, "Deep high-resolution representation learning for human pose estimation," in Proceedings of the IEEE Conference on Computer Vision and Pattern Recognition, pp. 5693-5703, IEEE, New York, NY, USA, June 2019. 
[7] H. S. Fang, S. Xie, Y. W. Tai, and C. Lu, "Rmpe: regional multiperson pose estimation," in Proceedings of the IEEE Conference on Computer Vision and Pattern Recognition, pp. 2334-2343, IEEE, San Juan, PR, USA, June 2017.

[8] Z. Cao, G. Hidalgo, T. Simon, S. E. Wei, and Y. Sheikh, "Openpose: realtime multi-person $2 \mathrm{~d}$ pose estimation using part affinity fields," 2018, http://arxiv.org/abs/1812.08008.

[9] B. Xiao, H. Wu, and Y. Wei, "Simple baselines for human pose estimation and tracking," in Proceedings of the European Conference on Computer Vision (ECCV), pp. 466-481, Springer, Munich, Germany, September 2018.

[10] M. Andriluka, U. Iqbal, E. Insafutdinov et al., "Posetrack: a benchmark for human pose estimation and tracking," in Proceedings of the IEEE Conference on Computer Vision and Pattern Recognition, pp. 5167-5176, IEEE, San Juan, PR, USA, June 2018.

[11] M. Munaro, S. Michieletto, and E. Menegatti, "An evaluation of $3 \mathrm{~d}$ motion flow and $3 \mathrm{~d}$ pose estimation for human action recognition," in Proceedings of the RSS Workshops: RGB-D: Advanced Reasoning with Depth Cameras, Berkeley, CA, USA, July 2013.

[12] S. Hadfield, K. Lebeda, and R. Bowden, "Natural action recognition using invariant $3 \mathrm{D}$ motion encoding," in Proceedings of the European Conference on Computer Vision, pp. 758-771, Springer, Glasgow, UK, September 2014.

[13] H. Rhodin, J. Spo"rri, I. Katircioglu et al., "Learning monocular 3D human pose estimation from multi-view images," in Proceedings of the IEEE Conference on Computer Vision and Pattern Recognition, pp. 8437-8446, IEEE, San Juan, PR, USA, June 2018.

[14] L. Yang, S. Li, D. Lee, and A. Yao, “Aligning latent spaces for $3 \mathrm{~d}$ hand pose estimation," in Proceedings of the IEEE International Conference on Computer Vision, pp. 2335-2343, IEEE, Cambridge, MA, USA, June 2019.

[15] A. Akash, M. Akhand, M. A. H. Akhand, and N. Siddique, "Robust face detection integrating novel skin color matching under variant illumination conditions," International Journal of Image, Graphics and Signal Processing, vol. 13, no. 2, pp. 1-15, 2021.

[16] Y. Wang, Y. Zhao, Z. Guo, M. Qi, Y. Fan, and H. Meng, "Diffusion Tensor image segmentation based on multi-atlas active shape model," Multimedia Tools and Applications, vol. 78, no. 24, Article ID 34231, 2019.

[17] H. Zhao, Y. Liu, and S. Li, "Online learning behavior based personality recognition," Open Education Research, vol. 25, no. 5, pp. 110-120, 2019.

[18] W. Cai and Z. Wei, "PiiGAN: generative adversarial networks for pluralistic image inpainting," IEEE Access, vol. 8, Article ID 48451, 2020.

[19] X. Ning, P. Duan, W. Li, and S. Zhang, "Real-time 3D face Alignment using an encoder-Decoder network with an efficient Deconvolution Layerfficient deconvolution layer," IEEE Signal Processing Letters, vol. 27, pp. 1944-1948, 2020.

[20] W. Cai and Z. Wei, "Remote sensing image classification based on a cross-attention mechanism and graph convolution," IEEE Geoscience and Remote Sensing Letters, vol. 10, pp. 1-5, 2020.

[21] X. Ning, K. Gong, W. Li, and L. Zhang, "JWSAA: Joint weak saliency and attention aware for person re-identification," Neurocomputing, vol. 453, pp. 801-811, 2020.

[22] W. Cai, B. Liu, Z. Wei, M. Li, and J. Kan, "TARDB-Net: tripleattention guided residual dense and BiLSTM networks for hyperspectral image classification," Multimedia Tools and Applications, vol. 1, pp. 1-22, 2021.
[23] X. Yu, J. Yang, and Z. Xie, "Training SVMs on a bound vectors set based on Fisher projection," Frontiers of Computer Science, vol. 8, no. 5, pp. 793-806, 2014.

[24] S. E. Wei, V. Ramakrishna, T. Kanade, and Y. Sheikh, "Convolutional pose machines," in Proceedings of the IEEE Conference on Computer Vision and Pattern Recognition, pp. 4724-4732, IEEE, Las Vegas, NV, USA, July 2016.

[25] Z. Cao, T. Simon, S. E. Wei, and Y. Sheikh, "Realtime multiperson $2 \mathrm{~d}$ pose estimation using part affinity fields," in Proceedings of the IEEE Conference on Computer Vision and Pattern Recognition, pp. 7291-7299, IEEE, Honolulu, HI, USA, July 2017.

[26] Y. Ding, J. Yang, J. Ponce, and H. Kong, "Homography-based minimal-case relative pose estimation with known gravity direction," in Proceedings of the IEEE Transactions on Pattern Analysis and Machine Intelligence, August 2020.

[27] X. Yu, F. Jiang, J. Du, and D. Gong, "A cross-domain collaborative filtering algorithm with expanding user and item features via the latent factor space of auxiliary domainsfiltering algorithm with expanding user and item features via the latent factor space of auxiliary domains," Pattern Recognition, vol. 94, pp. 96-109, 2019.

[28] Z. Wang, C. Zou, and W. Cai, "Small sample classification of hyperspectral remote sensing images based on sequential joint deeping learning modelfication of hyperspectral remote sensing images based on sequential joint deeping learning model," IEEE Access, vol. 8, Article ID 71353, 2020.

[29] W. Cai and Z. Wei, "Diversity-generated image inpainting with style extraction," 2019, http://arxiv.org/abs/1912.01834.

[30] Z. L. Yang, S. Y. Zhang, Y. T. Hu, Z. W. Hu, and Y. F. Huang, "Vae-Stega: linguistic steganography based on variational auto-encoder," IEEE Transactions on Information Forensics and Security, vol. 16, pp. 880-895, 2020.

[31] X. Ning, X. Wang, S. Xu et al., "A review of research on cotraining. concurrency and computation: practice and experience," Bio-Engineering Applications, vol. 32, Article ID e6276, 2021.

[32] Y. Kim and D. Kim, "A CNN-Based 3D human pose estimation based on projection of depth and ridge data," Pattern Recognition, vol. 106, 2020.

[33] M. Dantone, J. Gall, C. Leistner, and L. Van Gool, "Human pose estimation using body parts dependent joint regressors," in Proceedings of the IEEE Conference on Computer Vision and Pattern Recognition, pp. 3041-3048, IEEE, San Juan, PR, USA, June 2013.

[34] J. Su, A. X. Liu, Z. Sheng, and Y. Chen, "A partitioning approach to RFID identification," IEEE/ACM Transactions on Networking, vol. 28, no. 5, pp. 2160-2173, 2020.

[35] S. Johnson and M. Everingham, "Learning effective human pose estimation from inaccurate annotation," in Proceedings of the 24th IEEE Conference on Computer Vision and Pattern Recognition, pp. 1465-1472, IEEE, Colorado Springs, CO, USA, June 2011.

[36] J. Su, R. Xu, S. Yu, B. Wang, and J. Wang, "Idle slots skipped mechanism based tag identification algorithm with enhanced collision detection," KSII Transactions on Internet and Information Systems, vol. 14, no. 5, pp. 2294-2309, 2020. 